\title{
Ant Colony Algorithm for Solving the Multicast Routing Problem with QoS Constraints
}

\author{
DU Shengli ${ }^{1, a}$ \\ ${ }^{1}$ Academy of Information Technology, Luoyang Normal University,Luoyang,471022,China \\ aemail:dslhn@sina.com
}

Keywords: Multicast Routing Algorithm; Multicast Tree; Ant Colony Algorithms

\begin{abstract}
With the development of multimedia network, more and more real-time multimedia applications require a network capable of satisfying QoS constraints such as delay and bandwidth bound so that the messages reach each destination node at almost the same time within a certain specified time limit. Therefore, an Ant colony algorithm for solving multicast routing problem with QoS constraints is proposed. In the algorithm, group ants carry out searching separately and alternate information after one search. After a period of time, the rotary table is adapted to choosing a path and avoids stagnation, and the optimal one is selected from the paths by combining with multi-constrained QoS. Computer experiments show that the algorithm has a better performance and establish the multicast tree quickly.
\end{abstract}

\section{Introduction}

QoS multicast routing aims at meeting the QoS requirements of each business, and generates a minimum multicast spanning tree which includes all the multicast group members at the same time. Different businesses have different QoS constraints, such as bandwidth, delay, cost, and so on. The QoS routing problem is NP-complete problems, and the heuristic algorithm is much complex or too high in computational complexity. Aiming at this problem, ant colony optimization algorithm is used to solve the multicast routing problem at present. Ant colony optimization algorithm is a new optimization method proposed in recent years. The algorithm does not rely on mathematical description of the specific issues, but it has a strong global optimization performance and the parallelism. It has gotten better result in solving NP-complete problem such as the Traveling Salesman Problem. However, the search algorithm spends much more time and is easy to stagnate. The solution can not conduct a comprehensive search of space in the calculation process and easily leads to a large number of invalid searches or local optimum problems. In order to find a multicast tree, some people uses the ant colony algorithm to optimize path combinations based Dijkstra algorithm. However it wastes a lot of time when using greedy algorithm to find the path, and it is difficult to meet the requirements effectively. According to above, in order for the search of the optimal path and the establishment of the multicast tree which meets QoS constraints in multicast routing, a new ant colony algorithm is proposed in the paper. Experiments show that the algorithm has a better performance than the other routing algorithm and establish the multicast tree quickly.

\section{The Network Model and Problem Description}

Usually, $G=(V, E)$ stand for the network model. $V$ is the network nodes and $E$ is the network links . $T=(V 1, E 1) \in G$ is the current multicast tree.st $\in(V-V 1)$ is destination nodes which is defined by $\mathrm{D}$ to join the multicast group. $\mathrm{p}=\mathrm{p}(\mathrm{sc}, \mathrm{dt})$ is a selected path from the source node sc to the destination $\mathrm{dt}$, $\mathrm{e}$ is a link on the path of $\mathrm{p}(\mathrm{e} \in \mathrm{p})$. Each link of the tree $\mathrm{T}$ corresponds to the four positive real numbers $(\delta, \mathrm{c}, \mathrm{b})$. Among them, $\delta, \mathrm{c}, \mathrm{b}$ denote respectively the delay, cost and bandwidth of e. The problem with QoS constraints is showed as follows:

$$
\left\{\begin{array}{c}
\mathrm{D}(\mathrm{p})=\sum_{\mathrm{e} \in \mathrm{p}} \operatorname{delay}(\mathrm{e}) \\
\mathrm{B}(\mathrm{p})=\min \{\mathrm{b}(\mathrm{e})\} \\
\mathrm{C}(\mathrm{p})=\sum_{\mathrm{e} \in \mathrm{p}} \operatorname{cost}(\mathrm{e})
\end{array}\right.
$$


For the node $\mathrm{d}$ to join the tree, The multicast routing problem with QoS constraints is to find a path $\mathrm{p}(\mathrm{s}, \mathrm{d})$ to meet the following conditions:

$$
\left\{\begin{array}{c}
D(p) \leq D \\
B(p) \geq B \\
\min \{C(p)\}
\end{array}\right.
$$

Where D, B indicate respectively the multicast tree's delay constraints, bandwidth constraints. The ants' collectivity behavior reflects an information positive feedback phenomenon. The ant colony algorithm is a random search algorithm, which gets optimal solution through Simulating the process of ants' looking for food as shown in follow:

Step 1:intitializaton( $N c$ is iteration time, $k$ is ant number, $p$ is the transfer probability);

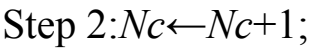

Step 3:kヒk+1;

step 4:if table is full then goto Step 3;

Step 5:calculting $p$, every ant is transferred from $i$ to $j$ according to $p$ probability and $j$ is inserted into table;

Step 6:if $k<$ the total number of ant $\mathrm{m}$ then goto Step 3;

Step 7:the total length of every ant going by is calculated separately and updating the information;

Step 8:if $N c<N c_{\max }$ then goto Step 2;

Step 9:return the shortest path;

Step 10:Halt.

\section{The Proposed Algorithm}

In this section, we will introduce our algorithm in detail. In the searching process our algorithm, two group ants carry out searching separately. One finds the optimal path from the source to destination, and the other finds path from the destination to source. After one search, they alternate information each other. All ants are assigned to two paths so as to avoid stagnating by choosing one. Meanwhile, in every searching process, the previous received optimal path and the probability of the path are saved. When each ant chooses the next node, the probability of previous search is introduced to speed up the search speed. After a period of time, ants choose the path according to rotary table, which can ensure diverse solution and avoid stagnation. The probability choice and rotary table choice carry on across. The probability choice is carried on several times and then the rotary table choice is carried on. The probability choice can protect the excellent solution and the rotary table choice can produce the opportunity of the better solution.

We firstly define the transfer formula of the state:

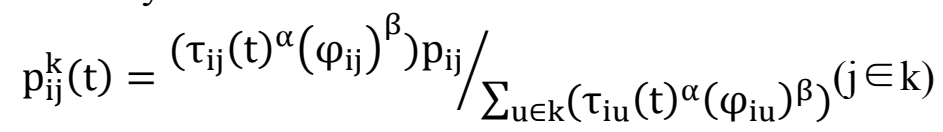

Where $\tau_{\mathrm{ij}}(\mathrm{t})$ is the information of the edge $(\mathrm{i}, \mathrm{j}), \alpha$ is the relative importance of the track, $\beta$ is the relative importance of visibility, pij is the probability of the edge $(\mathrm{i}, \mathrm{j})$ which is selected in the previous selection. $\mathrm{k}=\{0,1, \ldots, \mathrm{n}-1\}$ is the node set that ant $\mathrm{k}$ can choose next. The probability of previous search is must calculated, this makes the overall probability of the current search smaller, and does not meet the sum probability of every path full.

So the formula (3) is modified by $\mathrm{p}_{\mathrm{ij}}^{\mathrm{m}}(\mathrm{t})=\mathrm{p}_{\mathrm{ij}}^{\mathrm{k}}(\mathrm{t}) / \sum_{\mathrm{t} \in \mathrm{k}} \mathrm{p}_{\mathrm{it}}^{\mathrm{k}}(\mathrm{t})(\mathrm{j} \in \mathrm{k})$

The proposed algorithm is written by follow:

AntMultiRouting $(G, D, s, \delta, b)$

//Where, $G$ represents a network topology,$D$ is a set of destination nodes, $s$ is a source node, $\delta$ is an upper bound of end-to-end delay and $b$ is the minimum bandwidth constraint.

Input: a network topology $G=(V, E)$, a source node $s$, a set of destinations $D$, an upper bound of 
end-to-end delay $\delta$, a positive real number $\boldsymbol{b}$.

Output: a multicast tree $\mathrm{T}$ with the best multicast delay and bandwidth constraints.

Step 1 Initialize the network, input source node, destination node sets, $N c, \delta$ and $b$

Step 2 For each ant chooses the next node $j(j \in D)$ from the current node $i$ based on (4). Nodes $j$ will be inserted a queue named $t k$. So the next node which is chosen by the ant from destination to source is inserted into another queue named $t k_{2}$. After searching time, the path is searched by rotary table. The strategy makes the search constringency quickly in the beginning because the probability search is adopted, and makes the search diverse and avoids stagnation in the later part of the search because the rotary table search is adopted.

Step 2 According to $\tau_{\mathrm{ij}}(\mathrm{t}+1)=(1-\rho) \tau_{\mathrm{ij}}(\mathrm{t})+\sigma \tau_{\mathrm{ij}}, \sigma \tau_{\mathrm{ij}}=\sum_{\mathrm{i}=1}^{\mathrm{k}} \sigma \tau_{\mathrm{ij}}^{\mathrm{k}}$ modify the information amount of each path; $\mathrm{Nc} \leftarrow \mathrm{Nc}+1$;

Step 3 If $\mathrm{Nc} \geq \mathrm{Nc}_{\max }$ and $\left(\mathrm{tk}_{\mathrm{k}}=\mathrm{tk}_{2}=\Phi\right)$ then calculate $\mathrm{tk}$ and $\mathrm{tk}_{2}$ by formula (5):

$\mathrm{p}=\frac{\left(\mathrm{af}_{\mathrm{d}}+\mathrm{bf}_{\mathrm{dj}}+\mathrm{gf}_{\mathrm{b}}\right)}{\mathrm{C}(\mathrm{T}(\mathrm{s}, \mathrm{d}))}$

(Where $f_{d}=\emptyset_{d}(D(p(s, d))-D), f_{d j}=\emptyset_{d j}\left(p(p(s, d))-C_{j}\right), f_{b}=\emptyset_{b}(p(p(s, d))-B)$, $\emptyset_{\mathrm{d}}(\mathrm{U})=\left\{\begin{array}{c}1, \mathrm{U} \leq 0 \\ \mathrm{r}_{\mathrm{d}}, \text { otherwise }\end{array}, \emptyset_{\mathrm{dj}}(\mathrm{U})=\left\{\begin{array}{c}1, \mathrm{U} \leq 0 \\ \mathrm{r}_{\mathrm{dj}}, \text { otherwise }\end{array}, \emptyset_{\mathrm{b}}(\mathrm{U})=\left\{\begin{array}{c}1, \mathrm{U} \leq 0 \\ \mathrm{r}_{\mathrm{b}}, \text { otherwise }\end{array}.\right)\right.\right.$

In formula (5) $a, b, g$ respectively stands for the proportion of delay, cost and the bandwidth. $\Phi_{\mathrm{d}}(U)$ is the penalty function of delay. When the delay constrains satisfy $D(P(s, d)) \leq D$, it equal to 1 . Otherwise, its value is $r_{\mathrm{d}} \in(0,1)$. For $\Phi_{\mathrm{dj}}(U)$ and $\Phi_{\mathrm{b}}(U)$, the meaning is the same. All these values are set as $r_{\mathrm{d}}=r_{\mathrm{dj}}=r_{\mathrm{b}}=0.25$.

\section{Analysis of algorithm and computer simulation}

Give a network model which is eight nodes network model. Suppose the node $\mathrm{s}$ is the source node; the node $\mathrm{D}$ is the purpose node. The characteristic of the chain is described with four vectors (delay, cost, bandwidth). The QoS constraints are $\mathrm{b}=70, \delta=46, \mathrm{c}=10$, the coefficient is $\mathrm{a}=\mathrm{b}=\mathrm{g}$ $=1$. The experiment in this paper employs the modified Waxman to generate the network model .The model is very identical to the real network, and it is widely applied in the simulation experiments. According to given network, we define $\alpha$ and $\beta$ : $\alpha$ is the information factor,which means the relative importance of the track. It makes ants tend to choose the previous path along with increasing $\alpha . \beta$ is the heuristic factor, which means the relative importance of visibility. It is easy to make a local minimum while $\beta$ is smaller. On the other hand, it is slower in convergence while $\beta$ is larger. In the experiment we set $\alpha=0.3, \beta=1.5,|D|=20$. Different parameters' results are shown in table 1.

Table 1 The computation time of the algorithm.

\begin{tabular}{|c|c|c|c|}
\hline$\alpha$ & $\beta$ & Destination nodes & computation time $(\mathrm{ms})$ \\
\hline 0.1 & 3 & 24 & 15 \\
\hline 0.15 & 2 & 25 & 14 \\
\hline 0.3 & 1.5 & 20 & 10 \\
\hline 0.9 & 4 & 30 & 23 \\
\hline 0.85 & 6 & 32 & 27 \\
\hline 0.4 & 7 & 27 & 18 \\
\hline
\end{tabular}

We can see from table 1 that in search of optimal solutions for state transfer, the probability of the previous optimal path is introduced to find the optimal solution. So, the optimal path has a high probability to be selected and speeds up the search. At the same time, every constraint is fully considered in solving multi-constrained multicast routing QoS. It has reduced the cost compared with the mini delay algorithm despite of adding the delay, and it has reduced delay compared with the mini cost algorithm in spite of adding the cost. It can be seen also from the table that the algorithm has the better computing time. 


\section{Conclusion}

The main problem that we focused in this paper is to determine a multicast subnetwork that guarantees certain bounds including the delay and bandwidth bound from the source to all destinations. We propose and present this problem to solve the multi-constrained multicast routing QoS. Based on the basic ant colony algorithm, two group ants separately search the path and rotary table is used to avoid stagnation, and the searching probability is optimized. The previous search probability of the path is introduced in every search to speed up the search. The optimal path satisfies multi constrains although it increases delay compared with mini delay algorithm and increases cost compared with mini cost algorithm. However it has a higher integer performance. We would like to consider the link delay as a time variant function in our future work and in morerealistic network scenarios.

\section{References}

[1] D. W. Wall. Mechanisms for Broadcast and Selective Broadcast, PhD thesis, Stanford University, 1980.

[2] Oliveira C. A. S, Pardalos P. M. A survey of combinatorial optimization problems in multicast routing[J]. Computers and Operations Research, 2005, 32:1953-1981.

[3] A Heuristic Algorithm for Delay Delay-Variation Bounded Least Cost Multicast Routing [J]. IEEE 2nd International Advance Computing Conference,2010:261-266.

[4] L Kou,G Markowsky, L Berman.A fast algorithm for Steiner trees in graphs[J]. Acta Informatica,1981,15(2):141-145.

[5] Kewen Li,Shiyi Fang (2007-10). Anycast QoS routing algorithm balancing the network load based on random method[J]. Proceedings of the second international conference on intelligent information management systems and technology,2007(2):111-114

[6] Ran Min, Suixiang Gao, Xu Bao. A Multi-constrained QoS routing algorithm based on the Ant Colony Algorithm[J]. Computer engineering and application, 2005 (7):142 -144.

[7] Sahoo S P, Kabat M R, Sahoo A K. Tabu Search Algorithm for Core Selection in Multicast Routing[C]. International Conference on Communication Systems and Network Technologies, 2011:17-21. 